\title{
Static and Dynamic Accuracy of Vitreoretinal Surgeons
}

\author{
F. Peral-Gutierrez ${ }^{1}$, A. L. Liao ${ }^{2}$, C. N. Riviere ${ }^{2}$ \\ ${ }^{1}$ School of Engineering, University of Valladolid, Spain \\ ${ }^{2}$ The Robotics Institute, Carnegie Mellon University, Pittsburgh, Pa., USA
}

\begin{abstract}
Accuracy in manual manipulation in a simulated microsurgical environment has been studied. Three vitreoretinal microsurgeons completed a series of static and dynamic tasks. Results for overall rms position error, overall maximum position error, and estimated physiological tremor amplitude were calculated. The rms amplitude of the error vector in three dimensions averaged $166 \mu \mathrm{m}$ in a stationary pointing task, and 2009-4506 $\mu \mathrm{m}$ in the dynamic tasks. The maximum error vector magnitude during the stationary task averaged $408 \mu \mathrm{m}$ during the stationary tests, and 3531-6788 $\mu \mathrm{m}$ in the dynamic tasks. The rms amplitude of tremor during the stationary task averaged 76-101 $\mu \mathrm{m}$ in the three spatial coordinates, with an average vector magnitude of $156 \mu \mathrm{m}$ rms.
\end{abstract}

Keywords-Microsurgery, accuracy, robotics

\section{INTRODUCTION}

Human hand movement contains various involuntary components, including physiological tremor [1], jerk [2], and drift [3]. These components have limited performance in microsurgery, and cause certain types of procedures to be generally infeasible, such as retinal vein cannulation and arteriovenous sheathotomy [4]. Recent years have seen research toward suppression of these components in order to suppress erroneous movement during microsurgery, including telerobotics [2], the shared-control "steady-hand" approach [5], and a handheld instrument with active error control [6]. Evaluation of such systems requires a suitable performance baseline for unassisted surgeons with which to compare the performance of such devices. There continues to be a general lack of such data, particularly in dynamic tasks, in which the target is a trajectory rather than a fixed point.

Several one-dimensional (1-D) studies of motion have been reported for microsurgeons [1,7] and medical students [8]. Some studies have examined only physiological tremor $[1,8]$, the roughly sinusoidal component of involuntary motion. Others have examined also the general motion of surgeons [3,7]. Studies of the motion of microsurgeons in $3-\mathrm{D}$ are rare in the literature. An exception is [3]. However, in [3], surgeons attempted to hold a tool motionless without

Funding provided by National Science Foundation (grant no. EEC9731748) and National Institutes of Health (grant no. 1 R01 EB000526). a visually prominent target to allow easy monitoring of relative position, likely increasing the magnitude of drift.

The present paper presents preliminary results of an ongoing study quantifying the overall accuracy and physiological tremor amplitude of vitreoretinal surgeons in static and dynamic tasks in 3-D.

\section{Methodology}

The experiment was conducted using the ASAP optical tracking system [9] to record the three-dimensional position of the tip of the handheld instrument. ASAP uses an infrared (IR) light-emitting diode (LED) array, modulated at $5 \mathrm{kHz}$, to illuminate the workspace. A white delrin ball 4.7 $\mathrm{mm}$ in diameter is attached to the tip of the microsurgical instrument. The 3-D position of the ball is found using two 2-D position-sensitive detectors that receive the IR illumination reflected by the ball. The system performs synchronous detection and filtering to enhance the accuracy of the recordings. Data are recorded at $1500 \mathrm{~Hz}$ and downsampled to $150 \mathrm{~Hz}$.

The physical setup for the experiment simulated vitreoretinal microsurgery. A plaster face mask was placed around the illuminated workspace of ASAP. The inner surface or back side of the mask was covered with black light-absorbing paper to avoid reflection of IR by anything other than the marker ball. The mask, shown in Fig. **, was equipped with a rubber sclera (the white of the eye), with slots cut into it to simulate sclerotomies, small holes cut through the sclera through which vitreoretinal tools are inserted and which represent a fulcrum during surgical instrument motion.

The floor of the workspace was a small platform of black plastic. Through this platform were drilled 12 holes, evenly spaced around the circumference of a circle $5 \mathrm{~mm}$ in diameter, as shown in Fig. 2. Each hole was fitted with an optical fiber connected to a blue LED which could be turned off and on under the control of a computer. These LEDs identified the targets for the experiment.

The marker ball was painted white, and a red arc was marked with a pen, from the tip to the opposite side of the ball, where it meets the shaft of the instrument. As subjects held the instrument, they were instructed to use the point where the red line meets the horizon of the ball as the "pointer" for the experiment. ASAP tracks the centroid of the marker ball. To avoid artifacts due to the relatively large diameter of the ball, subjects were told to select a comfortable orientation of the red marker line with respect 
to a global fixed frame of reference, and to attempt to keep the red line in that same orientation throughout each trial. In other words, they were told to avoid rotating the instrument about its long axis. The optical fibers were $0.2 \mathrm{~mm}$ in diameter. Subjects were told to consider the center of each light spot to be the target. In the vertical direction (referred to here as $\mathrm{z}$ ), the subject was guided by the investigators to position the ball centrally in the workspace of ASAP, and was instructed to attempt to maintain that same elevation throughout the trial.

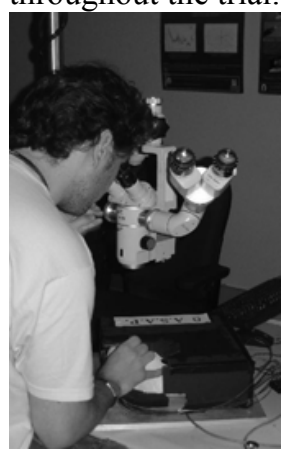

(a)

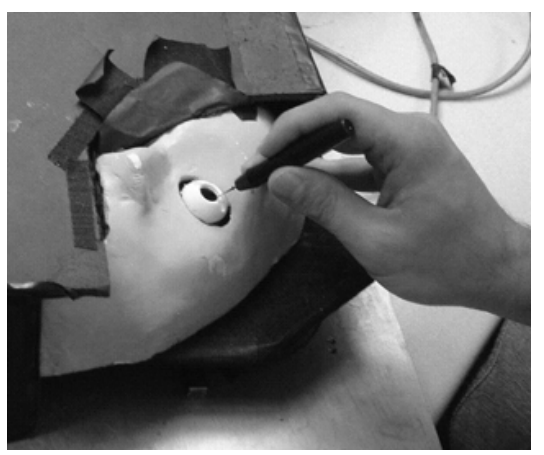

(b)
Fig. 1. (a) The experimental setup. (b) The facemask, showing the simulated sclerotomy with the handheld instrument entering through it.

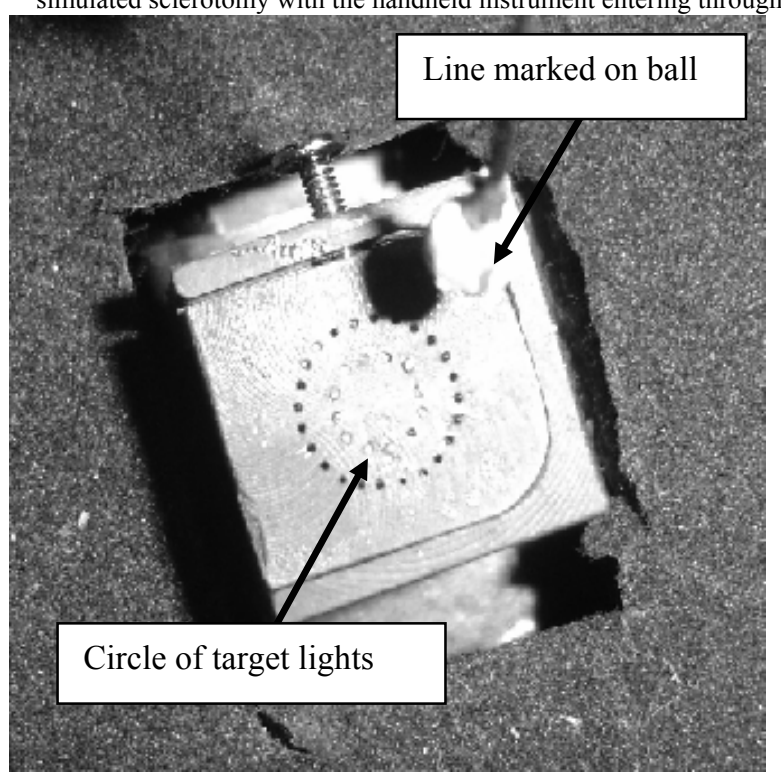

Fig. 2. The display for the target lights. An arrow indicates the 5-mmdiameter circle. The outer circle of dots was not used. The marker ball is visible at the tip of the instrument shaft at the upper right corner of the target platform. The line marked on the ball for use as a pointer is indicated with an arrow.

Three types of tests were conducted:

- Stationary. A single LED was illuminated for 20 $\mathrm{s}$, and subjects were instructed to point to it for the same duration. Each subject completed three tests.

- Tracking. The lights were turned on in succession so that the target moved around the circle at a constant rate, and the subject tracked the target. Subjects were instructed to interpolate between the discrete spots of light, moving in a smooth rather than a stepwise fashion, arriving at each light just as it turned on. Data were recorded for $2 \mathrm{~min}$. Each subject completed six tracking tests, consisting of clockwise and counterclockwise trials at each of the following periods of target motion around the circle: "slow" (1 min to travel around the circle), "medium" (30 s), and "fast" (15 s).

- Tracing. No lights were used. Subjects were instructed to trace the circle as in the tracking tests, but choosing a speed that was comfortable to them and that they felt was realistic for surgical manipulation. These tests lasted $20 \mathrm{~s}$. Each subject completed three tests.

Each subject therefore completed a total of 12 tests. The order of the 12 tests was randomized.

Root mean square error (rmse) was computed for each test, as well as the maximum error for each trial.

Physiological tremor frequency is often said to be roughly 8-12 Hz. Power spectra of all test results were visually examined to determine an approximate tremor band for the data from these tests. Based on this examination, the 6-12 $\mathrm{Hz}$ band was chosen. The rms amplitude of tremor in the stationary tests was estimated by passing the data through a sixth-order Butterworth bandpass filter with corner frequencies at 6 and $12 \mathrm{~Hz}$.

\section{RESUlts}

Table I presents the rmse for the stationary tests. For the nine trials of this type (three subjects, three trials per subject), the table shows the mean and standard deviation, as well as the results for the trial with the lowest rmse and the trial with the highest rmse. The table presents results for $x$, $y$, and $z$. The last row of the table presents the rms magnitude of the total 3-D error vector. Table II presents the total range, or maximum absolute value, of error for the stationary tests, presenting once again the mean, standard deviation, maximum, and minimum of the nine stationary trials.

Table III presents the rmse for the slow tracking tests, and Table IV presents the range or maximum error for these tests. Tables V and VI present the rmse and total range of error for the medium tracking tests. Tables VII and VIII present the corresponding results from the fast tracking tests. Clockwise and counterclockwise tests were analyzed together. Table IX presents the rmse for the tracing tests, while Table $\mathrm{X}$ presents the range of error.

Table XI presents the rms amplitude of tremor in the stationary tests.

Figure 3 presents a typical power spectrum. Figures 4 through 8 present typical results from the various types of tests conducted. 
TABLE I

RMS AMPLITUDE OF ERROR FROM THE STATIONARY TEST

\begin{tabular}{ccccc}
\hline Direction & mean $(\mu \mathrm{m})$ & $\begin{array}{c}\text { standard } \\
\text { deviation } \\
(\mu \mathrm{m})\end{array}$ & $\min .(\mu \mathrm{m})$ & $\begin{array}{c}\max . \\
(\mu \mathrm{m})\end{array}$ \\
\hline $\mathrm{X}$ & 91 & 67 & 19 & 209 \\
$\mathrm{Y}$ & 60 & 28 & 32 & 105 \\
$\mathrm{Z}$ & 88 & 163 & 10 & 520 \\
$\begin{array}{c}\text { Vector } \\
\text { magnitude }\end{array}$ & 166 & 152 & 41 & 537
\end{tabular}

TABLE II

OVERALL RANGE OF ERROR FROM THE STATIONARY TEST

\begin{tabular}{ccccc}
\hline Direction & mean $(\mu \mathrm{m})$ & $\begin{array}{c}\text { standard } \\
\text { deviation } \\
(\mu \mathrm{m})\end{array}$ & $\min .(\mu \mathrm{m})$ & $\begin{array}{c}\max . \\
(\mu \mathrm{m})\end{array}$ \\
\hline $\mathrm{X}$ & 408 & 214 & 124 & 735 \\
$\mathrm{Y}$ & 276 & 114 & 120 & 500 \\
$\mathrm{Z}$ & 323 & 579 & 50 & 1857 \\
$\begin{array}{c}\text { Vector } \\
\text { magnitude }\end{array}$ & 408 & 214 & 124 & 735
\end{tabular}

TABLE III

RMS AMPLITUDE OF ERROR FROM THE SLOW TRACKING TEST

\begin{tabular}{ccccc}
\hline Direction & mean $(\mu \mathrm{m})$ & $\begin{array}{c}\text { standard } \\
\text { deviation } \\
(\mu \mathrm{m})\end{array}$ & $\min .(\mu \mathrm{m})$ & $\begin{array}{c}\max . \\
(\mu \mathrm{m})\end{array}$ \\
\hline $\mathrm{X}$ & 1722 & 183 & 1471 & 1927 \\
$\mathrm{Y}$ & 1015 & 218 & 839 & 1314 \\
$\mathrm{Z}$ & 1420 & 747 & 541 & 2407 \\
$\begin{array}{c}\text { Vector } \\
\text { magnitude }\end{array}$ & 2009 & 179 & 1790 & 2310
\end{tabular}

TABLE IV

MAXIMUM ERROR FROM THE SLOW TRACKING TEST

\begin{tabular}{ccccc}
\hline Direction & mean $(\mu \mathrm{m})$ & $\begin{array}{c}\text { standard } \\
\text { deviation } \\
(\mu \mathrm{m})\end{array}$ & $\min .(\mu \mathrm{m})$ & $\begin{array}{c}\max . \\
(\mu \mathrm{m})\end{array}$ \\
\hline $\mathrm{X}$ & 3425 & 389 & 2901 & 4065 \\
$\mathrm{Y}$ & 2152 & 379 & 1884 & 2735 \\
$\mathrm{Z}$ & 3224 & 1293 & 1768 & 5021 \\
$\begin{array}{c}\text { Vector } \\
\text { magnitude }\end{array}$ & 3531 & 315 & 3250 & 4068
\end{tabular}

TABLE V

RMS AMPLITUDE OF ERROR FROM THE MEDIUM TRACKING TEST

\begin{tabular}{ccccc}
\hline Direction & mean $(\mu \mathrm{m})$ & $\begin{array}{c}\text { standard } \\
\text { deviation } \\
(\mu \mathrm{m})\end{array}$ & $\min .(\mu \mathrm{m})$ & $\begin{array}{c}\text { max. } \\
(\mu \mathrm{m})\end{array}$ \\
\hline $\mathrm{X}$ & 1832 & 243 & 1575 & 2149 \\
$\mathrm{Y}$ & 1745 & 161 & 1512 & 1962 \\
$\mathrm{Z}$ & 1697 & 472 & 1162 & 2409 \\
Vector & 2535 & 237 & 2300 & 2860 \\
magnitude & & & &
\end{tabular}

TABLE VI

MAXIMUM ERROR FROM THE MEDIUM TRACKING TEST

\begin{tabular}{ccccc}
\hline Direction & mean $(\mu \mathrm{m})$ & $\begin{array}{c}\text { standard } \\
\text { deviation } \\
(\mu \mathrm{m})\end{array}$ & min. $(\mu \mathrm{m})$ & $\begin{array}{c}\max . \\
(\mu \mathrm{m})\end{array}$ \\
\hline $\mathrm{X}$ & 4394 & 635 & 3711 & 5424 \\
$\mathrm{Y}$ & 4107 & 402 & 3712 & 4705 \\
$\mathrm{Z}$ & 4513 & 1296 & 3203 & 6150 \\
$\begin{array}{c}\text { Vector } \\
\text { magnitude }\end{array}$ & 4682 & 497 & 4162 & 5484
\end{tabular}

TABLE VII

RMS AMPLITUDE OF ERROR FROM THE FAST TRACKING TEST

\begin{tabular}{ccccc}
\hline Direction & mean $(\mu \mathrm{m})$ & $\begin{array}{c}\text { standard } \\
\text { deviation } \\
(\mu \mathrm{m})\end{array}$ & $\min .(\mu \mathrm{m})$ & $\begin{array}{c}\max . \\
(\mu \mathrm{m})\end{array}$ \\
\hline $\mathrm{X}$ & 2322 & 497 & 1851 & 2999 \\
$\mathrm{Y}$ & 1696 & 385 & 1197 & 2353 \\
$\mathrm{Z}$ & 2193 & 1015 & 705 & 3155 \\
$\begin{array}{c}\text { Vector } \\
\text { magnitude }\end{array}$ & 2880 & 602 & 2205 & 3812
\end{tabular}

TABLE VIII

MAXIMUM ERROR FROM THE FAST TRACKING TEST

\begin{tabular}{ccccc}
\hline Direction & mean $(\mu \mathrm{m})$ & $\begin{array}{c}\text { standard } \\
\text { deviation } \\
(\mu \mathrm{m})\end{array}$ & $\min .(\mu \mathrm{m})$ & $\begin{array}{c}\max \\
(\mu \mathrm{m})\end{array}$ \\
\hline $\mathrm{X}$ & 5573 & 1182 & 4073 & 7340 \\
$\mathrm{Y}$ & 4427 & 824 & 3222 & 5671 \\
$\mathrm{Z}$ & 5220 & 1925 & 2172 & 8012 \\
$\begin{array}{c}\text { Vector } \\
\text { magnitude }\end{array}$ & 5854 & 1057 & 4615 & 7392
\end{tabular}

TABLE IX

RMS AMPLITUDE OF ERROR FROM THE TRACING TEST

\begin{tabular}{ccccc}
\hline Direction & mean $(\mu \mathrm{m})$ & $\begin{array}{c}\text { standard } \\
\text { deviation } \\
(\mu \mathrm{m})\end{array}$ & $\min .(\mu \mathrm{m})$ & $\begin{array}{c}\max . \\
(\mu \mathrm{m})\end{array}$ \\
\hline $\mathrm{X}$ & 3916 & 302 & 3229 & 4258 \\
$\mathrm{Y}$ & 2206 & 226 & 1999 & 2538 \\
$\mathrm{Z}$ & 996 & 508 & 357 & 1574 \\
$\begin{array}{c}\text { Vector } \\
\text { magnitude }\end{array}$ & 4506 & 180 & 4080 & 4707
\end{tabular}

TABLE X

MAXIMUM ERROR FROM THE TRACING TEST

\begin{tabular}{ccccc}
\hline Direction & mean $(\mu \mathrm{m})$ & $\begin{array}{c}\text { standard } \\
\text { deviation } \\
(\mu \mathrm{m})\end{array}$ & $\min .(\mu \mathrm{m})$ & $\begin{array}{c}\max . \\
(\mu \mathrm{m})\end{array}$ \\
\hline $\mathrm{X}$ & 6717 & 843 & 5125 & 7641 \\
$\mathrm{Y}$ & 3708 & 347 & 3409 & 4250 \\
$\mathrm{Z}$ & 2689 & 1645 & 688 & 4789 \\
$\begin{array}{c}\text { Vector } \\
\text { magnitude }\end{array}$ & 6788 & 820 & 5135 & 7643
\end{tabular}

TABLE XI

RMS AMPLITUDE OF TREMOR FROM THE STATIONARY TEST

\begin{tabular}{ccccc}
\hline Direction & mean $(\mu \mathrm{m})$ & $\begin{array}{c}\text { standard } \\
\text { deviation } \\
(\mu \mathrm{m})\end{array}$ & $\min .(\mu \mathrm{m})$ & $\begin{array}{c}\max . \\
(\mu \mathrm{m})\end{array}$ \\
\hline $\mathrm{X}$ & 101 & 0.95 & 100 & 103 \\
$\mathrm{Y}$ & 90 & 0.47 & 89 & 91 \\
$\mathrm{Z}$ & 76 & 0.84 & 75 & 78 \\
Vector & 156 & 1.2 & 155 & 158 \\
magnitude & & & &
\end{tabular}

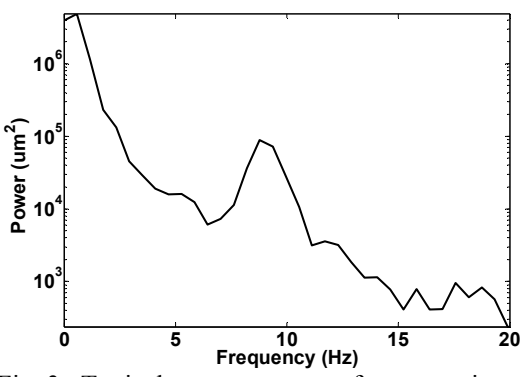

Fig. 3. Typical power spectrum from a tracing test. 


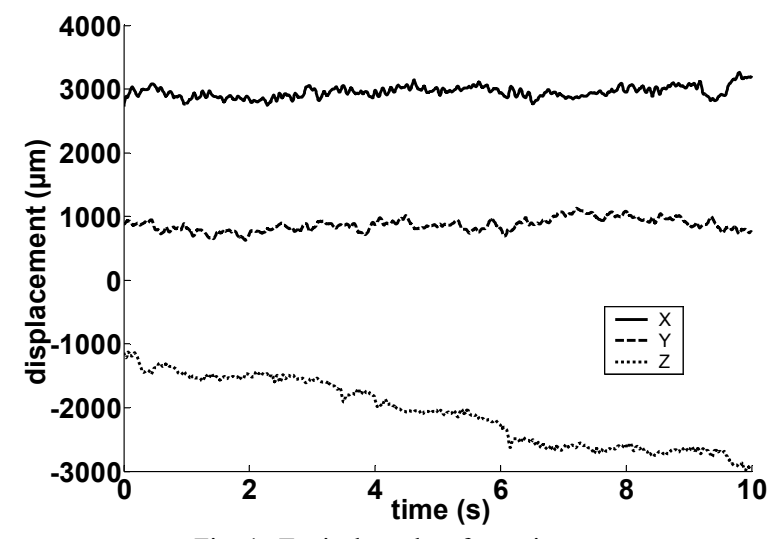

Fig. 4. Typical results of a stationary test.

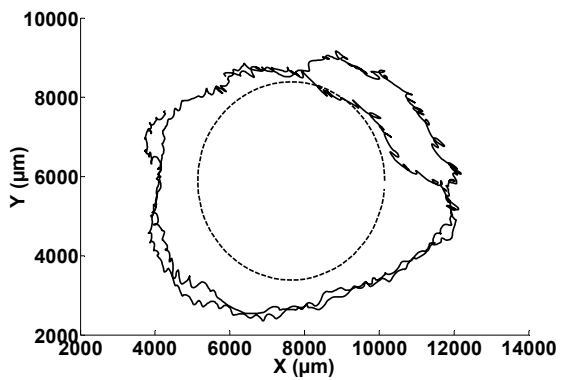

Fig. 5. Typical results of a slow tracking test.

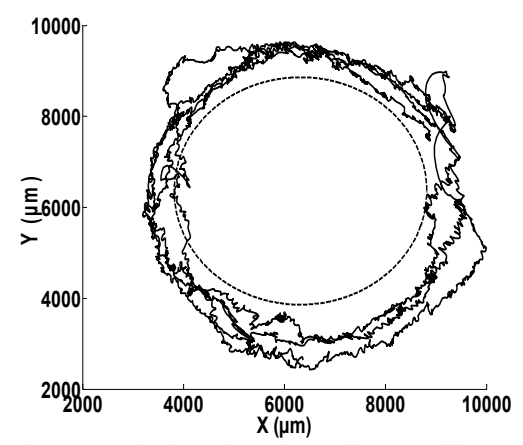

Fig. 6. Typical results of a medium tracking test.

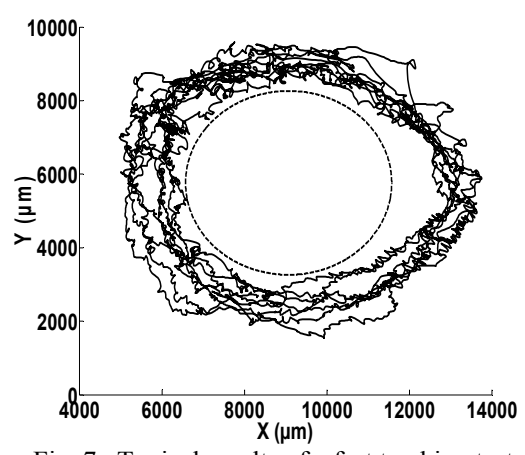

Fig. 7. Typical results of a fast tracking test.

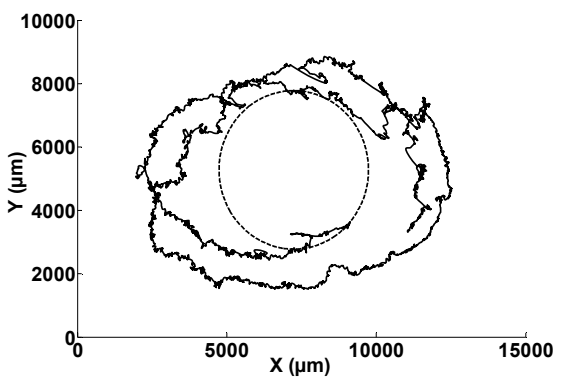

Fig. 8. Typical results of a tracing test.

\section{Discussion}

The tremor values reported in Table XI are comparable to those obtained during clinical microsurgery [10]. In the dynamic tasks (tracking and tracing), while some trials show relatively accurate results, as in Fig. 6, several trials exhibit tracing or tracing curves that are too large, as in Figs. 5 and 8. This may be due to partial occlusion of the viewing field by the marker ball and the interaction of this effect with the stereo view provided by the operating microscope. Further investigation is needed to determine whether the effect is due to this or some other cause.

\section{REFERENCES}

[1] R. C. Harwell and R. L. Ferguson, "Physiologic tremor and microsurgery," Microsurgery, vol. 4, pp. 187-192, 1983.

[2] P. S. Schenker, E. C. Barlow, C. D. Boswell, H. Das, S. Lee, T. R. Ohm, E. D. Palug, G. Rodriguez, and S. T. Charles, "Development of a telemanipulator for dexterity enhanced microsurgery," Proc. $2^{\text {nd }}$ Int. Symp. Med. Rob. Comput. Assist. Surg., pp. 81-88, 1995.

[3] L. Hotraphinyo and C. N. Riviere, "Three-dimensional accuracy assessment of eye surgeons," Proc. $23^{\text {rd }}$ Annu. Conf. IEEE Eng. Med. Biol. Soc., Istanbul, Oct. 25-28, 2001, pp. 3458-3461.

[4] W. N. Tang and D. P. Han, "A study of surgical approaches to retinal vascular occlusions," Arch. Ophthalmol., vol. 118, pp. 138-143, 2000

[5] R. Taylor, P. Jensen, L. Whitcomb, A. Barnes, R. Kumar, D. Stoianovici, P. Gupta, Z. Wang, E. de Juan, Jr., and L. Kavoussi, "A steady-hand robotic system for microsurgical augmentation," Int. J. Robot. Res., vol. 18, pp. 1201-1210, 1999.

[6] C. N. Riviere, W. T. Ang, and P. K. Khosla, "Toward active tremor canceling in handheld microsurgical instruments," IEEE Trans. Rob. Autom., vol. 19, pp. 793-800, 2003.

[7] C. N. Riviere and P. K. Khosla, "Characteristics of motion of eye surgeons," Proc. $19^{\text {th }}$ Annu. Conf. IEEE Eng. Med. Biol. Soc., Chicago, Oct. 30-Nov. 2, 1997.

[8] E. G. Walsh, "Physiological finger tremor in medical students and others," Neurol. Dis. Ther., vol. 30, pp. 63-78, 1994.

[9] L. Hotraphinyo and C. N. Riviere, "Precision measurement for microsurgical instrument evaluation," Proc. $23^{\text {rd }}$ Annu. Conf. IEEE Eng. Med. Biol. Soc., Istanbul, Oct. 25-28, 2001, pp. 34543457. 